\title{
Filigrane
}

Écoutes psychanalytiques

\section{En passer par les mots : places, interlocution et discours en entrevue}

\section{Irène Krymko-Bleton}

Volume 28, numéro 1, 2019

URI : https://id.erudit.org/iderudit/1064601ar

DOI : https://doi.org/10.7202/1064601ar

Aller au sommaire du numéro

Éditeur(s)

Santé mentale et société

ISSN

1192-1412 (imprimé)

1911-4656 (numérique)

Découvrir la revue

Citer cet article

Krymko-Bleton, I. (2019). En passer par les mots : places, interlocution et discours en entrevue. Filigrane, 28(1), 123-148.

https://doi.org/10.7202/1064601ar
Résumé de l'article

L’article présente les possibilités offertes par la pragmatique linguistique et la rhétorique pour l'analyse des entrevues de recherche. À l'aide d'exemples, l'article montre comment, en passant par la forme du discours et les façons de dire, il est possible de dégager le sens du propos, du contenu implicite au contenu inconscient de la parole du sujet adressée au chercheur. Traverser les mots convoque aussi le passage par des assignations de places au chercheur et à l'interlocuteur qui lui parle : échange conversationnel où la pensée s'élabore. 


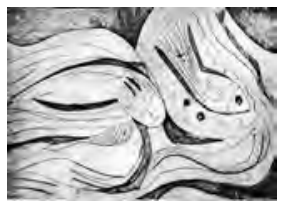

\title{
En passer par les mots: places, interlocution et discours en entrevue
}

\author{
Irène Krymko-Bleton
}

\begin{abstract}
Résumé: L'article présente les possibilités offertes par la pragmatique linguistique et la rhétorique pour l'analyse des entrevues de recherche. À l'aide d'exemples, l'article montre comment, en passant par la forme du discours et les façons de dire, il est possible de dégager le sens du propos, du contenu implicite au contenu inconscient de la parole du sujet adressée au chercheur. Traverser les mots convoque aussi le passage par des assignations de places au chercheur et à l'interlocuteur qui lui parle: échange conversationnel où la pensée s'élabore.
\end{abstract}

Mots clés: analyse du discours; recherche psychanalytique; pragmatique; rhétorique.

\begin{abstract}
This article considers the possibilities offered by linguistic pragmatics and rhetoric for the analysis of research interviews. Using examples, the article shows how it is possible, through the form of the discourse and the ways of saying, to disentangle the meaning of the discourse, from the implicit content to the unconscious content of the subject's speech addressed to the researcher. Travelling through words also calls for the passage through the assignment of places for the researcher and the interlocutor who speaks to him: a conversational exchange where thought is developed.
\end{abstract}

Key words: discourse analysis; psychoanalytic research; pragmatics; rhetoric.

es mots. Des phrases. La parole du sujet. Elle n'a plus tellement la cote aujourd'hui. Dans les années 70, nous nous insurgions contre la simplificatrice conception de la communication comme transfert de l'information entre émetteur et récepteur; que dire aujourd'hui à l'époque où l'image semble occuper toute la scène et où l'on communique par émoticons et emoji? Répondant à une lettre de condoléances, une dame, pourtant plus que septuagénaire, répond par ce message: « (4) \$. Comment l'interpréter? Les mots lui manquent? Ces emoji, que veulent-ils dire? Le deuxième est énigmatique. Le quatrième indique la colère, mais contre qui est-elle fâchée? En l'occurrence, contre son enfant décédé à cause de son intrépidité? 
L'outil du psychanalyste, c'est la parole. Même ceux qui travaillent avec les enfants d'avant la parole et doivent «entendre avec leurs yeux ${ }^{1}$ » s'adressent aux enfants avec des mots choisis avec le plus grand soin. La linguistique étudie la langue et, dans sa branche pragmatique, l'usage de la parole dans différentes situations interlocutives. Bien entendu, la philosophie et toutes les autres sciences humaines attachent de l'importance aux choix des expressions justes et au travail avec ou sur la langue. Je vais limiter néanmoins mon propos ici à la démonstration d'une collaboration possible entre deux disciplines, aujourd'hui peu prisées - psychanalyse et sciences du langage» - dans le cadre de recherches d'orientation psychanalytique à l'université. Toutes deux aident à pénétrer le labyrinthe de ces échanges qui déterminent notre existence individuelle et la coexistence collective.

\section{Le dire}

Les mots articulent notre pensée, disent, mais aussi dissimulent, mentent, opèrent... Shakespeare met dans la bouche de ses personnages des expressions qui visent à enlever aux mots leur puissance: "It is a kind of good deed to say well; and yet words are not deeds.» fait-il dire au Roi dans Henry VIII (acte III, scène II); dans Roméo et Juliette (acte II, scène II), Juliette se demande "What's in a name? A rose by any name would smell as sweet.» Pourtant, rien à faire. Il montre aussi que les noms, qui sont des mots autour desquels se construit l'identité sociale de chacun ou les expressions qui ont une puissance performative particulière, ne peuvent malgré tout être réduits à de simples sons. Le grand maître des Templiers, qui maudit du haut de son bûcher la famille des Capétiens jusqu'à la treizième génération ${ }^{2}$, se fie à leur force et la suite de l'histoire de France semble confirmer l'efficacité de son anathème. Plus prosaïquement: lorsqu'un juge prononce la sentence, les conséquences en sont immédiates; lorsqu'un pape prononce l'excommunication, elle a la force d'un acte; le prêtre qui baptise un enfant exécute une action symbolique qui donne la place à cet enfant dans l'univers des croyants. Ceux qui occupent les places les autorisant socialement à prononcer les paroles-actes ou ceux qui articulent des discours visant l'adhésion de masses ne s'expriment pas par des émoticons. Ils savent néanmoins qu'aujourd'hui le dire doit être court. Dans certaines situations, cela a toujours été: l'ordre «Feu !» est d'une remarquable efficacité, prononcé par une personne autorisée dans une situation définie. Quand on manque de mots ou que notre interlocuteur est insaisissable et qu'on passe aux gestes, de plus en 
plus souvent on prend un fusil ou on se suicide - les conditions de l'autorisation et du cadre ne sont alors nullement respectées.

Bien sûr, les mots se présentent rarement seuls; quand ils le sont, leur signification dépend largement du ton et du contexte. Organisés en phrases, elles-mêmes constitutives de différentes formes de discours, les mots forment la parole permettant l'échange interlocutif. Sa forme la plus courante est la conversation (dont l'entrevue de recherche fait partie). Celle-ci n'a éveillé l'intérêt de linguistes et de philosophes du langage qu'assez récemment, mais toutes les étapes qui, de proche en proche, ont mené à son étude nous intéressent.

\section{Convergences: entre la linguistique, la philosophie du langage et la psychanalyse, la recherche psychanalytique à l'université}

La linguistique moderne est née de travaux de Ferdinand de Saussure ${ }^{3}$, qui introduisit l'étude de la langue "en elle-même» et "pour elle-même», la fondant sur une conception du signe constitué d'un signifiant (une image acoustique) et de son signifié (un concept). Le signifiant et le signifié sont séparés par une barre horizontale. Bien que séparés, ils ne peuvent toutefois pas être disjoints; en revanche, le référent, la chose désignée, est, lui, extérieur au signe.

Nous retrouverons ce schéma, sensiblement modifié, chez Jacques Lacan. Je vais y revenir.

Bien que direct héritier de Saussure, le structuralisme a élargi l'étude de la langue en abordant les questions du langage. Ainsi, réfléchissant aux diverses fonctions du langage, Roman Jakobson attirait l'attention sur sa fonction phatique, dont l'importance pour nos analyses de données sera soulignée plus bas. Elle s’incarne dans des énoncés qui servent à vérifier si la communication passe bien entre celui qui parle et ceux qui l'écoutent ( "vous me suivez?», «c'est compris?», etc.). Pour Jakobson, elle fait même partie des six grandes fonctions du langage: «Il y a des messages qui servent essentiellement à établir, prolonger, ou interrompre la communication, à vérifier que le circuit fonctionne, à attirer l'attention de l'interlocuteur ou à s'assurer qu'elle ne se relâche pas...» (Jakobson, 1963, p. 217)

Après les grammairiens Jacques Damourette et Édouard Pichon, au milieu du xx siècle, Émile Benveniste (1902-1976) introduit dans le champ de la linguistique le principe d'une prise en compte de la subjectivité des interlocuteurs engagés dans la communication interpersonnelle. Avec lui s'ouvre l'étude de la parole dans une relation intersubjective mise en 
contexte. La brèche qui s'élargit ainsi progressivement dans la conception purement formaliste de l'étude de la langue culmine dans les années 70 par l'introduction de la notion d'inconscient par des linguistes psychanalystes et les chercheurs intéressés par l'analyse de discours ${ }^{4}$.

C'est néanmoins le philosophe du langage $\mathrm{H}$. Paul Grice qui a proposé le premier un modèle d'analyse de conversation à partir du principe qui stipule qu'une conversation est bien plus que la somme de chacune des interventions que les interlocuteurs y ont faites. En effet, parallèlement à la linguistique, la philosophie poursuivait de son côté sa réflexion sur le langage, en le questionnant à partir d'une de ses formes, extrême, la logique. Un contemporain de Saussure, Charles S. Pierce s'est aussi intéressé au signe (chez lui constitué de trois éléments: le representamen, l'objet et l'interprétant). L'étude de l'interprétant s'autonomisera, à terme, dans la pragmatique, qui nous sert de référence dans notre travail de recherche. À son tour, Ludwig Wittgenstein allait remarquer que le sens n'est pas seulement endogène au mot (les définitions du dictionnaire) mais institutionnellement produit. Il a introduit la notion de "jeux du langage», en insérant dans l'étude des échanges interlocutifs, leurs contextes et leurs déroulements. Ainsi, élève de Wittgenstein, le philosophe anglais John Langshaw Austin allait différencier des types d'effets produits par chaque énoncé en contrastant notamment les énoncés qui servent à décrire le monde (constatifs) à ceux qui réalisent une action (performatifs). Ainsi, dire «il promet» est un constatif mais «je promets » un performatif, engageant la personne qui l'a formulé. Ouvrir une séance formelle de $\mathrm{CA}$, promettre quelque chose, baptiser quelqu'un ou jeter un anathème sont en effet des actes (performatifs), mais qui ne peuvent être effectifs que sous certaines conditions fortement contraignantes. «La séance est levée», énoncé par le mécanicien qui vient de changer vos pneus, est de l'humour, alors que c'est la seule manière de clore officiellement la séance de débats dans une assemblée délibérante.

Toute énonciation constitue un acte locutoire, puisqu'il situe le locuteur dans le langage - qu'il se déclare ouvert à l'interlocution ou, au contraire, qu'il interdise toute réciprocité. Les actes illocutoires instituent un jeu et l'interlocuteur doit tenir compte de ses règles (fût-ce en les ignorant, en ne les comprenant pas, en les pervertissant - comme dans quelques exemples plus bas). Les actes illocutoires ont des conditions de réussite: pour réussir, ils dépendent de conventions, du contexte d'échange, de l'attitude ou de la capacité de l'interlocuteur de saisir à la fois le contenu sémantique et la nature instituante de l'acte. 
Les effets perlocutoires de l'énonciation, prévus ou imprévus, impliquent l'incidence produite dans le for intérieur de l'interlocuteur. Ils auront des répercussions sur son tour de parole, sa participation au jeu interlocutif. En incluant l'effet effectivement produit, l'acte perlocutoire introduit de l'incertitude. Cet effet peut en effet être congruent ou non avec l'intention de celui qui parle, dépendre de nombre d'éléments contextuels du côté du locuteur - bonne ou mauvaise évaluation des circonstances, des codes culturels de l'interlocution, de sa maîtrise du langage ou de la rhétorique, de sa capacité d'accomplir ou non ce qu'il a promis de faire lorsqu'il s'agit d'une promesse ou d'une menace... Du côté de l'interlocuteur, la perlocution laisse entrevoir la possibilité du malentendu. Comme au départ de cette rencontre de recherche:

L'entrevue commence par un malentendu linguistique. Il me dit: vous avez fait un long voyage depuis (pays d'origine de la chercheure)? Je suis surprise de sa question, car il sait que je vis au Québec, on avait parlé de mon immigration au téléphone. En fait, il parlait de voyage en sens psychologique. Il avait bien compris que j'avais immigré. Cela augure un peu de la suite de l'entretien, car j'ai tendance à penser qu'il ne comprend pas ce que je lui dis. Parfois c'est le cas. Mais comme dans le cas du mot «voyage», c'est aussi souvent moi qui ne comprends pas ce qu'il me dit, du moins au premier abord. (laboratoire Irène Krymko-Bleton)

Il s'agit donc de l'effet psychologique ressenti par l'interlocuteur et cet effet ne dépend pas uniquement de l'intention de celui qui parle, mais tout autant de la façon dont son interlocuteur reçoit sa parole, qu'elle soit explicite ou qu'elle nécessite un calcul interprétatif.

L'étude du fonctionnement du langage amène à comprendre l'échange conversationnel comme un jeu dont une partie des règles est imposée, extralinguistiquement, avec une force plus ou moins contraignante. Par exemple, la rencontre psychanalytique, basée sur l'échange verbal, est aussi une transaction. S’y croisent donc les ajustements dans la langue (tutoiement ou vouvoiement?) et les conditions de son énonciation qui en constituent le cadre. Il en est de même avec une entrevue de recherche.

Le psycholinguiste contemporain Frédéric François fait un pas de plus. Son expérience de recherche, impliquant la parole des enfants, l'amène à renverser les perspectives dégagées par les philosophes du langage qui l'avaient précédé à propos des échanges dialogiques. Elle le rapproche singulièrement 
de la position tenue par les psychanalystes: c'est la parole de l'autre qui est à l'origine de la parole de soi. À la suite du théoricien russe de la littérature Mikhail Bakhtine (1895-1975) qui avait développé les concepts de dialogisme et de polyphonie dans le récit, il enseigne que l'enfant n'est pas fondamentalement dans la relation avec la langue, mais avec la parole des autres. Est-ce différent pour les adultes? Les psychanalystes pensent que non.

La psychanalyse et les théorisations du langage évoluent et certains de leurs courants se rapprochent. À preuve, personne ne s'offusque aujourd'hui lorsque le philosophe Francis Jacques, dans l'étude des liens entre langage et subjectivité écrit: "Au lieu de tenir la subjectivité pour première, ou à l'opposé de la tenir pour nulle et non avenue, j'ai déplacé le primat sur la relation interpersonnelle.» (Jacques, 1982, p. 12)

Le travail de la cure considéré comme une co-construction entre analysant et analyste rencontre ici la conception de l'échange langagier conçu comme la résultante «de la constitution réciproque des participants à l'œuvre conjointe du sens» (Jacques, 1982, p. 32), ce qui est une version optimiste d'une co-construction coopérative. Si l'«œuvre du sens» se fait à deux, côté locuteur, la parole varie en fonction de la représentation que le sujet se fait de son interlocuteur et des réactions de celui-ci à ce qui est dit et, côté interlocuteur, l'interprétation du dit est fonction non seulement du cadre de l'échange et de la représentation que le sujet se fait du locuteur qu'il entend (et qu'il voit), mais aussi des intentions qu'il lui suppose...: autant d'occasions de voir s'installer le malentendu, ce qui constituerait la version pessimiste de cette co-construction coopérative.

Nous rencontrons les premiers croisements entre la linguistique et la psychanalyse chez les grammairiens Damourette et Pichon. Ce dernier est psychanalyste. Jacques Lacan leur doit ses développements sur la dénégation et la forclusion. Mais une deuxième rencontre entre la psychanalyse et la linguistique est manquée. Lacan, s'inspirant de Saussure, reprend bien le schéma où signifiants et signifiés sont séparés par une barre horizontale et sont extérieurs à leurs référents, mais il les disjoint en leur dessinant des destinées indépendantes. Les linguistes ne se sont guère reconnus dans cette appropriation des idées saussuriennes. Émile Benveniste, convoqué par Lacan pour garantir quand même la reconnaissance de son système, en conçoit une idée très critique. À partir de ce moment, Lacan ne va plus parler de la linguistique mais de ses «linguisteries».

La psychanalyse n'a évidemment pas pour objet le langage comme tel; ce sont les manifestations de l'inconscient qui l'intéressent. Le psychanalyste 
au travail, tout autant que le chercheur - selon l'école à laquelle il appartient, ses intérêts et ses choix -, peut tenir compte d'une multitude d'indices non linguistiques qui se dégagent dans une situation d'interlocution. L'apparence de l'interlocuteur, son ton, ses réactions physiques, ses comportements ou ses mimiques sont nécessairement pris en compte, même de façon involontaire, dans toute situation d'échange direct. Les manifestations de l'inconscient passent toutefois massivement dans l'usage de la parole, surtout dans le cadre de la cure, qui a principalement le langage comme matériau de travail. Comme l'écrit la linguiste Claudine Normand, ce que le sujet a à dire, «le sujet le dit précisément, dans les contraintes de la langue, par elles et/ou malgré elles» (Normand, 1985, p. 125). Toute image que l'on donne de soi, comme toute parole, est produite sur le fond de codes qui les encadrent. Ces codes changent selon les sociétés, les époques, les usages. La concordance ou la discordance de ces indices avec les codes en vigueur participent à ce que les sociologues de l'école de Chicago ont nommé «la présentation de soi».

Parfois, les diverses manifestations extraverbales de la présentation de $\mathrm{soi}^{5}$ rendent l'écoute de la parole plus difficile. Lorsqu'il s'agit d'un texte transcrit, elles sont manquantes; en revanche, le texte (ici, la transcription des entrevues) offre l'avantage de pouvoir être travaillé aussi longtemps et minutieusement que nécessaire.

Quelles que soient ses relations avec la linguistique, revenons à Lacan, et à son Rapport au Congrès de Rome en 1953 ( «Fonction et champ de la parole et du langage en psychanalyse. Partie I. Parole vide et parole pleine dans la réalisation psychanalytique du sujet»). Il parlait alors de "parole du patient»:

Qu'elle se veuille agent de guérison, de formation ou de sondage, la psychanalyse n'a qu'un médium: la parole du patient. L'évidence du fait n'excuse pas qu'on le néglige. Or toute parole appelle réponse.

Nous montrerons qu'il n'est pas de parole sans réponse, même si elle ne rencontre que le silence, pourvu qu'elle ait un auditeur, et que c'est là le cœur de sa fonction dans l'analyse. [... l'art de l'analyste doit être de suspendre les certitudes du sujet, jusqu'à ce que s'en consument les derniers mirages. Et c'est dans le discours que doit se scander leur résolution.

Quelque vide en effet qu'apparaisse ce discours, il n'en est ainsi qu'à le prendre à sa valeur faciale: celle qui justifie la phrase de Mallarmé quand il compare l'usage commun du langage à l'échange d'une monnaie dont l'avers comme l'envers ne montrent plus que des figures effacées et que 
l'on se passe de main en main «en silence». Cette métaphore suffit à nous rappeler que la parole, même à l'extrême de son usure, garde sa valeur de tessère ${ }^{6}$.

Même s'il ne communique rien, le discours représente l'existence de la communication; même s'il nie l'évidence, il affirme que la parole constitue la vérité; même s'il est destiné à tromper, il spécule sur la foi dans le témoignage.

Aussi bien le psychanalyste sait-il mieux que personne que la question y est d'entendre à quelle "partie» de ce discours est confié le terme significatif, et c'est bien ainsi qu'il opère dans le meilleur cas: prenant le récit d'une histoire quotidienne pour un apologue qui à bon entendeur adresse son salut, une longue prosopopée pour une interjection directe, ou au contraire un simple lapsus pour une déclaration fort complexe, voire le soupir d'un silence pour tout le développement lyrique auquel il supplée. (Lacan, 2014, p. 245-250)

Qui pourrait mieux dire?

\section{La parole du sujet}

Comme l'indiquent Élisabeth Roudinesco et Michel Plon dans le Dictionnaire de la psychanalyse, le vocable de «sujet» est apparu dans le vocabulaire psychanalytique lorsque l'inconscient a été théorisé par Jacques Lacan dans la relation du sujet au signifiant. Cette théorisation est issue de la prise en compte des effets de la relation langagière dans laquelle est plongé le petit d'homme dès avant sa naissance. Le terme n'apparaît pas dans le Vocabulaire de la psychanalyse de Laplanche et Pontalis.

[...] c'est en 1960, dans «Subversion du sujet et dialectique du désir dans l'inconscient freudien», que Lacan s'appuyant sur la théorie saussurienne du signe linguistique, énonce sa conception de la relation du sujet au signifiant: «Un signifiant c'est ce qui représente le sujet pour un autre signifiant». (Roudinesco, 1997, p. 1030)

Le sujet désormais, donc. Non seulement le sujet des grammairiens, mais le sujet de l'inconscient ${ }^{7}$. Dès lors, c'est à travers le langage que la recherche universitaire d'orientation psychanalytique peut se mettre à la recherche du sujet. Si Freud avait bien découvert que l'inconscient se faufilait dans les anfractuosités du discours comme les lapsus ou autres glissements de 
langue, depuis, pragmatique linguistique et rhétorique offraient alors aux psychanalystes intéressés une plus large panoplie d'outils pour repérer le sujet en suivant ses façons de dire. Les études de linguistes et de philosophes du langage ont ouvert la voie à l'étude des façons de parler choisies sciemment ou non. Au-delà de la généralité selon laquelle elles apparaîtraient immanquablement lors de conflits psychiques, les linguistes-psychanalystes ont identifié certaines des formes langagières qui se manifestent lorsque des mécanismes de défense ${ }^{8}$ sont à l'œuvre. L'analyse de toutes ces façons de dire permet donc de discerner les intentions des interlocuteurs et ouvre aussi l'accès aux conflits sous-jacents, plus au moins conscients ou tout à fait inconscients, de celui qui parle.

En guise de première reconnaissance du terrain, faisons un bref survol avec L'art du sous-entendu (2018) de Laurent Pernot, qui introduit le lecteur dans le monde des manipulations discursives de ces orateurs qui visent à orienter l'écoute de leurs interlocuteurs pour obtenir les résultats argumentatifs escomptés. Dans sa préface, il souligne l'omniprésence des sous-entendus dans la masse des entendus de tout discours, de tout échange de paroles. Une grande partie de ces sous-entendus relève d'ailleurs de notre éducation et de notre culture. Selon le milieu dans lequel nous vivons, nous sommes introduits très tôt aux codes qui le régissent et nous rend au mieux maladroits, au pire aveugles, aux codes et aux usages d'autres cultures ou d'autres milieux que nous connaissons mal. Par exemple, dans certains milieux québécois, il est impoli de manifester ouvertement son désir - il faut donc comprendre que «ça ne me dérange pas» est en fait le substitut de «je le souhaite». Dans bien des sociétés occidentales, le reproche "vous n'auriez pas dû», énoncé lorsque l'on reçoit un cadeau, est en fait un remerciement. Il faut vraiment être Japonais ou très bien connaître le Japon pour savoir si l'acquiescement d'un interlocuteur plus jeune à ce que dit le plus âgé est une vraie adhésion à ses idées ou n'est qu'un signe de politesse obligatoire. Sous toutes les latitudes, la politesse (dont les règles visent à préserver la face des interactants) oblige souvent à dire le contraire de ce que l'on pense; cela n'empêche nullement deux interlocuteurs partageant le même code d'effectuer le calcul interprétatif adéquat et d'insinuer ainsi réciproquement leurs vraies intentions en se sachant compris.

La rhétorique enseigne qu'il y a au moins deux niveaux dans la construction d'un discours. Le premier, qui vise à exprimer l'intention globale du locuteur, concerne l'intégralité du propos. À l'intérieur de ce discours global, les phrases ou les mots ponctuent certains moments où apparaissent les thèmes particuliers: ceux-ci peuvent avoir été planifiés, mais aussi introduits 
de façon non-intentionnelle, voire inconsciente. Les constructions volontaires, intentionnelles, plus au moins réussies, côtoient donc ces échappées de la langue qui signalent des confusions, des émotions, des idées inconscientes se frayant un chemin sous divers déguisements.

L'analyse d'un propos procède donc par un va-et-vient entre le propos dans sa globalité et les détails dont il est constitué, mais aussi entre des manières directes de dire et des manières indirectes. Allégorie, ironie, atténuation, hyperbole, synecdoque, métalepse, épanorthose...: on discerne dans les langues européennes quelques 300 figures de style qui permettent une très grande diversité des jeux possibles auxquels se prête la langue. Or, si les arts du discours s'étaient perfectionnés pour des professionnels du verbe depuis l'Antiquité, leur usage n'est pas réservé aux seuls orateurs ou politiciens. C'est en tout cas ce que révèlent les textes d'entrevues auxquels la recherche d'orientation psychanalytique a affaire.

\section{Analyse de discours et recherche d'orientation psychanalytique}

Leur parole devrait donc nous mener à la rencontre de nos sujets - dans les deux sens du mot (!). Lacan lui-même y invite en parlant de la psychanalyse en tant qu'agent de la formation ou de sondage. En fait, pour mettre à jour le sens des propos d'un sujet, psychanalyse, pragmatique linguistique et rhétorique convergent, avancent alors de concert. Ce sens qui, souvent, lui échappe mais aussi qui s'élabore au fur et à mesure que le sujet parle au chercheur qui l'écoute.

Certes, l'expérience clinique du chercheur l'a rendu sensible à certaines façons de dire; elle lui sert globalement de référence à l'écoute d'une entrevue de recherche. Aussi le recours à la pragmatique linguistique et aux leçons de la rhétorique peuvent pallier le manque ou l'insuffisance d'une telle expérience chez l'étudiant-chercheur. Toutefois, même pour le clinicien d'expérience, la référence à ces deux disciplines sert d'appui sérieux pour expliciter ses intuitions et préciser sa démarche interprétative.

\section{Dégager le sens}

La recherche qui prend en compte le langage et la forme qu'un sujet donne à son discours, nous plonge - comme le formule Frédéric François, qui décrit ainsi la recherche qualitative - dans des méandres, à savoir celles

[...] du dit, du non-dit, du difficile à dire. Des désirs, des interdits, des inquiétudes. Très peu de dit dans la masse du vécu. Encore moins de réfléchi 
dans la masse du dit ou du dicible. Du «conscient» et du l'«inconscient», quelle que soit la difficulté qu'on ait à donner à cette opposition un sens assignable. (François, 2005, p. 11)

Les énoncés d'un sujet circonscrivent un contenu, expriment des sentiments, mettent en jeu des relations avec son interlocuteur dans une recherche incessante d'équilibrage de places avec le chercheur, traduisant la position subjective implicite du sujet et campant ainsi la situation discursive.

Dans les années 70, la conception du langage proposée par Lacan guide François Flahault dans ses recherches sur le discours - «C'est la relation aux mots d'autrui qui fait la réalité du langage.» (Flahault, 1975, p. 136) Flahault construit une grille de lecture qui mobilise dans tout échange plusieurs registres de parole. Il prend en compte le contexte immédiat de l'échange, le contexte institutionnel et sociétal dans lequel cet échange se déroule et les types de discours mobilisés par les interlocuteurs. Ce qui le conduit à proposer une méthode d'analyse de discours cherchant à identifier les "places» que les interlocuteurs négocient pour eux et entre eux dans chaque échange de parole. Cette négociation est largement implicite dans l'échange; mais, mise en lumière, elle permet l'interprétation de la relation intersubjective des locuteurs, y compris au niveau inconscient. Des stratégies performatives dans la langue révèlent quelle place un locuteur souhaite occuper par rapport à l'autre en se situant par rapport à lui et en tentant de le maintenir à une place complémentaire à la sienne. Cette analyse en répartition de places permet une interprétation à la fois plus juste et plus nuancée de l'échange, voire des enjeux inconscients qui s'y nouent. Les propositions de Flahault servent de référence à toutes les analyses de discours effectuées dans mon laboratoire, le rapport de places remplaçant ici la référence à la relation transférentielle.

Le processus d'analyse d'une entrevue de recherche conduit vers le moment où s'impose au chercheur l'impression de comprendre de quoi il s'agit effectivement dans les propos d'un sujet. Il commence à pouvoir identifier les indices convergents à partir desquels il peut mettre le doigt sur le sens (éventuellement caché) de la parole de son interlocuteur.

\section{À la recherche du contenu implicite et inconscient}

Je ne m'attarderai pas ici aux méthodes qui s'arrêtent à l'analyse du contenu explicite d'une entrevue. De telles analyses, communes à toutes les recherches, comprennent plusieurs étapes: identification de thèmes, identification des logiques d'enchaînements thématiques... Dans la méthode qui 
est la mienne, ces thèmes ne sont pas traités comme des faits objectifs, mais comme des tentatives d'élaboration subjective à trois partenaires: le sujet de recherche annoncé, la personne qui a accepté de répondre en entrevue et la personne qui mène l'entrevue. Ce sont ces tentatives d'élaboration qui vont devenir le centre d'intérêt du chercheur. L'analyse des façons de dire devient du coup une sorte de pivot autour duquel s'organise en grande partie le travail.

Dans tous les cas, le chercheur note la progression du discours de son sujet. Il cherche à savoir si les procédés rhétoriques utilisés lui permettent la reprise du discours et sa progression (qui participent donc à l'élaboration de la pensée) ou s'ils semblent inefficaces, traduisant ainsi une perturbation importante. Les fils associatifs sont-ils repris ou non, mènent-ils vers une expression plus ample, plus satisfaisante, ou non?

Voici comment la chercheure Marie-Lorrain Pradelles-Monod présente une telle élaboration qui s'opère tout au long d'une entrevue qu'elle analyse:

Ce motif transparaît dans la façon dont elle construit les sources de sa remémoration. Nous en avons souligné les différents modes: il y a ce à quoi elle réfléchit tout en parlant, ce à quoi elle adhère au fur et à mesure qu'elle l'énonce, ce qu'elle élabore comme certitudes ou incertitudes, ce qu'elle considère comme probable, ce à quoi elle donne d'emblée le statut d'un souvenir. Cependant, quels que soient ces modes, ils sont parcourus par un mouvement identique: donner une assise à sa remémoration. (Pradelles-Monod, 1999, p. 282)

Les associations propres du chercheur qui résultent de sa connaissance de l'ensemble de l'échange - autant dans son déroulement que dans son contexte - permettent d'augmenter progressivement sa compréhension de ce que dit le sujet qui lui parle, y compris des éléments voilés, dissimulés, voire énigmatiques. Le chercheur est donc co-producteur du sens. Un autre chercheur pourrait partir sur une autre piste associative et proposer une construction mettant l'accent sur un aspect différent du discours du sujet. La coopération interprétative ne s'arrête d'ailleurs pas là, puisque ces deux chercheurs hypothétiques auraient, eux aussi, à s'entendre entre eux quant à l'essentiel du propos et de la position du sujet. Leur compréhension du sujet s'en trouverait ainsi enrichie.

Les exemples qui suivent visent à illustrer ce qui précède. Ce sont de petits extraits issus de recherches de mon laboratoire, des analyses effectuées 
par des chercheurs dans le cadre de leurs recherches universitaires présentées dans des articles; occasionnellement, ils réferrent à la vie courante. Ces extraits exhibent la mise en jeu de la relation entre sujet et chercheur qui campe la rencontre, ils déchiffrent l'expression de sentiments, ils éclairent le contexte des propos tenus et clarifient leur finalité, ils mettent en lumière les signes de mécanismes de défense qui apparaissent à certains moments de l'entrevue.

Si certains exemples insistent sur les détails de formulation, en général, le chercheur n'a pas besoin de conduire une analyse aussi formelle pour l'ensemble du texte de chacune de ses entrevues - ce qui serait très onéreux en temps. En préparant le texte pour l'analyse approfondie, il aura remarqué des moments lui semblant plus parlants que d'autres. D'ailleurs, nous ne lisons aucun livre, même de grande qualité littéraire, avec une attention également constante. Certains énoncés nous parlent, d'autres nous ennuient. Ils nous intéresseront peut-être lors d'une autre lecture, quand notre analyse pourra nous y amener; ou nous allons les laisser de côté.

De quelle nature sont les énoncés que nous retenons? Ne sont-ils pas, précisément, dans certains cas, ceux à valence perlocutoire? Pourquoi tant d'éléments du discours sont-ils passés sous silence dans l'analyse finale et pourquoi tel énoncé vient-il définir le locuteur et inspirer le destinataire? Ce que cherche le lecteur, ici le psychologue, l'objet de sa «drague», terme emprunté à Barthes, c'est l'extrait, le fragment, la phrase qui pourra l'inspirer. Le terme "drague», polysémique contient les deux idées: «aller à la pêche» et «être séduit». (Rebourg-Roestler, 2005/6, p. 675)

À partir de quel moment une entrevue nous parle-telle? Qu'est-ce qui nous amène à sentir qu'elle prend pour nous un sens précis? Voilà des questions déterminantes que tout chercheur devrait se poser. Rien ne s'oppose néanmoins à une procédure inverse: que le chercheur procède, en quelque sorte, à l'aveugle. Il peut choisir au hasard n'importe quel extrait du texte, le soumettre à une analyse minutieuse et en tirer des questions ou des hypothèses interprétatives qu'il lui faudra étoffer et vérifier en reprenant l'ensemble du texte, après que celui-ci eut été situé dans le contexte de la situation de l'entrevue. Toutefois, proposons ici de montrer comment se repèrent des éléments qui, assez classiquement, interpellent la lecture et sont de bons candidats pour organiser le discours.

La recherche déjà citée de Pradelles-Monod effectuée auprès de trois générations de femmes d'une même famille (la grand-mère, la mère et la 
fille) - la quatrième génération (l'arrière-grand-mère) étant à l'origine de l'installation de la famille en France - met à jour le processus de l'intégration de la famille en France et repère les traces de liens avec le pays d'origine.

C'est une intuition forte, proche de la surface thématique, qui tout d'abord impose à la chercheure le rôle primordial de la référence à l'axe temporel et à ses moments saillants. Ce qui la conduit ensuite à repérer dans ce registre les points qui organisent le discours, à identifier des mots clés et enfin à exposer explicitement pour ses lecteurs le caractère structurant du registre temporel dans l'organisation du discours de la mère:

Cette analyse met en évidence, dans la construction de son histoire, une organisation autour de deux points temporels: la naissance de sa mère, la mort de sa grand-mère. Il y a un "avant» enclos entre la naissance de sa mère et la mort de sa grand-mère et un «après» cette mort. Ces deux événements font «origine». (Pradelles-Monod, 1999, p. 283)

\section{Le repérage de rapport de places}

Outre le repérage de mots clés et la vérification de l'hypothèse qu'ils sont bien tels, l'analyse peut partir à la recherche d'indices de tentatives faites par les interlocuteurs pour modifier ou instaurer un rapport de places entre eux. Le sujet participant à une recherche peut recourir à plusieurs procédés langagiers pour établir ou modifier le rapport de places entre lui et le chercheur. Les expressions phatiques ${ }^{9}$ en font partie; pour autant souvent on ne peut en discerner le sens que si des éléments non verbaux peuvent être analysés. Le ton et, bien sûr, le contexte constitué par l'ensemble du propos et la situation de l'entrevue doivent donc être pris en considération. Dans un texte de Jakobson publié sur le site Roseau pensant, les auteurs du site ${ }^{10}$ rappellent que les acteurs du théâtre de Stanislavski pouvaient évoquer quelques dizaines de situations différentes en énonçant simplement les mots «ce soir»:

Dans le cadre des recherches que nous avons entreprises [...] sur la description et l'analyse du russe courant contemporain, nous avons demandé à cet acteur de répéter l'épreuve de Stanislavski. Il nota par écrit environ cinquante situations impliquant toutes cette même phrase elliptique et enregistra sur disque les cinquante messages correspondants. La plupart des messages furent décodés correctement et dans le détail par des auditeurs d'origine moscovite. (Jakobson, s. d., s. p.) 
Ce n'est donc pas dans les mots, mais dans la façon de les dire que se trouvait la clé de la compréhension du message. Dans les recherches impliquant des entrevues, le chercheur dispose d'un enregistrement dont il peut se servir si nécessaire, même s'il se concentre sur le texte luimême. Lorsqu'on ne dispose que du verbatim d'une entrevue, l'intonation manque. Dans un échange courant, comme dans une séance d'analyse, ces aspects ne sont pas disjoints. Néanmoins, lors d'une analyse purement textuelle, le contexte (de la phrase, de la situation générale, du moment de l'échange) permet d'attribuer des sens différents à un même mot. Voici donc, à titre d'exemple, une même expression phatique - «vous me suivez?»- qui acquiert bien des sens différents selon le contexte. Parfois les modifications apportées à cette formulation rendent assez explicite le sous-entendu qu'elle abrite.

Dans la situation 1, dans le cadre d'une recherche sur la paternité, un père brosse un tableau un peu incohérent des difficultés qu'il rencontre à se faire reconnaître comme personne responsable par la mère de ses enfants et la famille de celle-ci - qui tentent de lui retirer le droit de visite. Il raconte comment il s'occupe bien de ses enfants. Il termine un long discours, plutôt raisonnable, au sujet de l'éducation des enfants par:

Tu parles doucement, faut que tu fasses comprendre à l'enfant que tu comprends, vous me suivez un petit peu là?

C'est le « un petit peu là » qui met la puce à l'oreille en signant la position que le sujet tente d'occuper par rapport à la chercheure. Il ne vise pas ici de s'assurer que la chercheure l'écoute, mais lui montre sa supériorité dans l'art de s'occuper des enfants. Il tente de rabaisser la chercheure, ce qui, par ailleurs, est une des manifestations compensatoires des sentiments d'impuissance et d'agressivité qu'il éprouve et pour lesquels il ne trouve pas d'issue. Face à une femme, la chercheure, cette petite phrase incite à aller jusqu'à se demander si elle n'indique pas la complexité du mode de relation qu'il établit avec les femmes en général. Le répondant participe à la recherche de son plein gré et on peut penser qu'en acceptant l'entrevue, il est venu demander qu'on le trouve raisonnable et digne de confiance. Mais il vient aussi de révéler à quel point il manque de discernement.

Dans la situation 2, le style de parole et le contexte de l'exposé indiquent aussi la tentative d'établir une relation où le sujet pourrait se sentir supérieur et compenser pour le sentiment d'humiliation qu'il ressent (et qu'il 
s'emploiera à nier tout au long de l'entrevue). Ce sujet, immigrant dont les diplômes ne sont pas reconnus à leur juste valeur, s'interrompt au milieu d'un assez long propos où il expose une sorte de philosophie constituée de vérités de La Palice (signe de banalisation et de mise à distance de ce qui pourrait le toucher) et demande à la chercheure: "Vous me suivez?»

Et enfin un troisième exemple: dans une recherche sur l'accouchement prématuré, le père d'un enfant qui a connu des complications dont l'explication lui est difficile demande à la chercheure «Vous me suivez?», inquiet qu'il est de ne pas s'exprimer assez clairement pour qu'elle puisse se rendre compte de la difficulté de la situation. Ici c'est le ton et le regard, notés par la chercheure, qui indiquent la nature de la question. «Vous me suivez?», qui implique donc le chercheur, pourrait bien se dire aussi «Vous comprenez?», en déplaçant encore davantage cette inquiétude du côté du chercheur: c'est de lui et de ses capacités de comprendre qu'il s'agit.

En supposant que tout texte, toute expression, puisse être polysémique, le chercheur a des chances de s'approcher au plus près de la vérité du sujet. Il s'assure de l'exactitude de ses interprétations en poursuivant l'analyse et en cherchant les indices concordants et discordants avec ceux déjà identifiés. Muni de la loupe pragmatique et rhétorique, le chercheur devient un lecteur Sherlock Holmes puisqu'une entrevue transcrite est devenue texte. Certes, cette mutation peut s'avérer appauvrissante si l'expression corporelle et les modulations de la voix pendant l'entrevue n'ont pas été notées. En revanche, il peut aussi arriver qu'elles se soient tellement imposées en entrevue à l'attention du chercheur que, une fois les analyses du texte entamées, il lui arrive de découvrir avec surprise combien le langage corporel non seulement ne concordait pas avec le verbe, mais le contredisait. L'air enjoué de quelqu'un qui raconte des blagues peut parfaitement dissimuler des sentiments dépressifs et un contenu lourd sous cet habillage comique.

Si l'on s'en tient aux seules paroles notées dans le verbatim, comment repérer les actions verbales par lesquelles le sujet cherche à influencer le chercheur? Jakobson parlait de la fonction conative du langage pour nommer les actes langagiers qu'un locuteur entreprend pour influencer son interlocuteur. Y servent notamment les déterminants de personne (par exemple, en français, le choix entre «tu» et "vous») et les déterminants temporels («maintenant», «avant», etc.).

Christine Rebourg-Roestler, en rendant compte de sa recherche sur les procédés rhétoriques chez des patients présentant une organisation perverse de personnalité, donne plusieurs exemples de telles tentatives. En analysant 
les réponses aux planches de Rorschach, elle note, dans l'exemple qui suit, l'usage de l'épanorthose ${ }^{11}$ :

Planche III (enquête) : «Vousproposez des personnages ici... Si vous connaissiez l'étymologie du mot... Personnage, de persona, qui fait allusion au simulacre car persona en latin c'est le masque... Vous auriez renoncé à cette proposition et préféré des êtres humains... Le vous le dis pour votre pratique, c'est une suggestion bien sûr!» (sourire) ${ }^{12}$.

La chercheure commente:

Dans cet exemple les déictiques «vous», «je» et «ici» situent clairement les deux protagonistes de l'acte d'énonciation et structurent l'environnement spatio-temporel, ce qui rend possible l'activité discursive elle-même. Le locuteur, par des modalités méta-linguistiques, revient sur la définition du mot «personnage» pour dévaloriser, de manière implicite, le destinataire dans son savoir. (Rebourg-Roestler, 2005/6, p. 678)

Toujours à propos de la passation de tests projectifs, Odile Hussein rapporte divers exemples de formulations qui visent à impliquer l'examinateur et le faire sortir de sa neutralité.

«Est-ce que je peux éventuellement mettre mes lunettes?» demande un sujet à la planche VI. Ce "éventuellement» invite l'examinateur à se défendre de l'idée qu'il pourrait sadiquement dénier cette possibilité au sujet (voir Dreyfus et Husain, 2001, p. 54).

La fonction conative (donc l'effort pour influencer l'interlocuteur) s'exprime de la façon la plus directe par les impératifs.

Dans une recherche sur les accouchements prématurés, un futur père, qui a tenu à ce que la rencontre avec la chercheure ait lieu dans son bureau à lui, commence la rencontre en lui ordonnant: "Asseyez-vous là! J'ai 40 minutes à vous donner». On apprend par la suite que tout dans sa vie semble menacé d'écroulement: il risque de perdre son entreprise, à la maison tout tourne autour des besoins de sa femme alitée et personne ne prend en compte ses besoins. Il compense donc son sentiment de désarroi pendant un court moment en endossant le rôle du patron et en attribuant à la chercheure celui d'une subalterne. Peut-être aussi tente-t-il de contrôler la pression qui monte et qui pourrait le faire éclater (en sanglot? de colère?). 
Un équilibrage des positions respectives entre les interlocuteurs se poursuit tout au long des entrevues, de façon plus au moins subtile et plus au moins consciente de part et d'autre. Il est bien entendu qu'un même sujet dans une même entrevue peut tenter d'occuper plusieurs places par rapport au chercheur et que le chercheur peut, éventuellement, procéder de la même manière. La recherche de la complicité, de la connivence ou de l'approbation peut coexister avec des tentatives de contrôle ou d'emprise; la camaraderie avec la condescendance; la déférence avec l'arrogance ou la dérision, etc. Dans un autre article (Krymko-Bleton, 2014) auquel le présent fait suite, j'avançais que l'analyse de la relation de places dans la situation de recherche remplace la notion de transfert dans une démarche thérapeutique. En effet, elle éclaire tout ce qui est dit dans la rencontre. Les motifs, le plus souvent inconscients, incitent les sujets à vouloir participer à la recherche et à nous parler. Ils s'adressent aux chercheurs, mais leur façon de parler trahit la présence de bien d'autres personnages derrière leur dos: qui sommesnous lorsqu'on recherche notre complicité, notre autorité? Lorsqu'on nous montre son savoir ou son innocence? ...

\section{Comment s'organise le discours du sujet}

Une fois les positions respectives du sujet et du chercheur repérées et donc une fois le cadre de la rencontre établi, notre intérêt se porte sur la façon dont les thèmes abordés se disent et se suivent. Le sujet prend-il de la distance par rapport à ce dont il parle ou, au contraire, semble-t-il envahi par les idées qui lui viennent? Comment s'en sort-il? Quels moyens linguistiques utilise-t-il pour garder un équilibre qui doit lui permettre de dire ce qu'il est venu communiquer au chercheur? Son discours est-il clair? S'empêtre-t-il dans des phrases qui ne trouvent pas leur fin? Peut-il se reprendre? Se permet-il d'exprimer des émotions ou s'expriment-elles malgré lui? Se commet-il lorsqu'il parle ou se cache-t-il dans un discours tissé de vérités communes et de clichés? En général, quel est son style?

On s'attardera ici sur une stratégie consistant à créer une distance entre le sujet et son dit. Comme elle prend de multiples formes discursives, les indicateurs auxquels le chercheur a à faire sont variés.

Par exemple, en soulignant ou en laissant entendre que ce qu'il avance n'a rien d'original puisque «tout le monde» le pense, ou lorsqu'il s'exprime en phrases toutes faites, le sujet tente de se désengager émotivement de ses propos. «C'est un cadeau du ciel», dit le père d'un enfant très prématuré pour nier les sentiments négatifs qu'il éprouve à l'égard de cet enfant. S'interdire 
de réfléchir de façon personnelle offre un refuge, fait rechercher la facilité du «sens commun», ses lieux communs, ses stéréotypes, ses clichés.

La répétition ou la réitération apparaissent très fréquemment pour marquer l'intensité des émotions soulevées, quelle que soit la diversité des sentiments (positifs ou négatifs) ainsi soulignés et quel que soit le degré d'intensité des événements dont l'importance est ainsi signalée. Elles traduisent fréquemment un essai de contrôler l'émotion et offrent au chercheur la possibilité de repérer la charge émotionnelle à travers cette expression langagière. Le sujet répète ce qu'il a déjà dit avec ou sans modification, comme dans «Elle l'a fait. Elle l'a vraiment fait!».

Dans sa recherche sur le trouble obsessionnel, Fabrice Choquette relève les moments de perturbation émotionnelle:

Chez un des sujets, elle rapporte une répétition sans modification à la planche IV :

Je vois deux têtes d'ours, deux têtes d'ours;

La réponse d'un autre sujet est une répétition avec modification: à la planche VII «Deux femmes ou deux jeunes êtres féminins».

La répétition peut aussi révéler un débordement émotionnel, une sidération, comme celle qui apparaît dans cette réitération avec modification à la planche II «Puis en bas, des souliers, deux souliers, des souliers de femme, des chaussures...» (Choquet, 2001, p. 205)

Pradelles-Monod relève dans une de ses entrevues la répétition insistante à propos d'un secret de famille:

Je sais pas//je sais pas elle en avait peut-être honte//enfin je sais pas euh elle voulait pas que je sache que ma mère n'était pas la vraie fille à mon grandpèrel/je sais pas//je sais pas//je sais pas (Pradelles-Monod, 1999, p. 312)

Les expressions visant à diminuer l'impact présumé de la parole énoncée constituent elles aussi de bons indices, comme «ça nous a ébranlés un tout petit peu». Le sujet peut, par projection, anticiper la réaction du chercheur: «Vous pensez que c'est important, mais je n'ai pas été bien impressionné».

Sera-t-on surpris de constater, non pas dans des entrevues de recherche fondées sur la singularité d'une parole mais dans les exemples proposés par la banque de l'Office de la langue française pour illustrer la définition de l'euphémisme («expression atténuée d'une idée ou d'un fait dont 
l'évocation directe pourrait déplaire ou choquer ${ }^{13}$ »), que l'évolution des normes linguistiques sociétales au Québec nous interdit dorénavant de parler des «aveugles», terme remplacé par celui de «non-voyants» (être aveugle est-il offensant?), ou que l'expression «vieux» a disparu, remplacée par les «personnes du troisième âge» ou les «aînés »? Mot qui, incidemment, suggère que la société dans son ensemble serait devenue fraternelle: ce vocable d' «aînés» fonde en effet l'illusion que nous serions tous une grande famille, la métaphore (de la société comme famille) laissant croire que cette famille serait en plus fraternelle, sans mère ni père. L'analyse des verbatim québécois nous oblige désormais à tenir compte de cette évolution sociétale du langage.

Nombre de lieux communs se fondent d'ailleurs sur une métaphore, ce qui permet de cacher une charge affective. Dans l'entrevue d'une participante à une recherche sur l'avortement provoqué, qui n'avait pas informé sa mère du sien, apparaît la métaphore «mère poule», qui caractérise la mère qui «couve» trop ses enfants. En l'occurrence, la participante évite de dire ouvertement qu'elle trouve sa mère abusive et intrusive. Cette métaphore sert d'atténuation désindividualisante, place la relation mère-fille sur un terrain moins personnel, plus général, celui de l'ensemble des filles pourvues de «mères poules».

Parmi les procédés les plus courants, qui signent des tentatives de mise à distance par la dépersonnalisation du propos - autant dans les entrevues que dans le langage courant - on retrouve l'usage des pronoms «on» et «ça».

Le caractère impersonnel du pronom «on» peut être renforcé par l'usage du verbe "croire», à l'exemple de Choquet: "on croit percevoir», comme si la perception qui s'impose pouvait prêter au doute.

Une des interlocutrices de Pradelles-Monod, en se servant de «ça» présente ainsi la rencontre de son père et de sa mère: «ils se sont connus comme ça», "c'est comme ça qu'ils se sont connus». Sa propre rencontre avec son futur mari emprunte le même chemin «ils étaient toute une bande de copains $[. .$.$] et puis voilà c'est comme ça qu'on s'est retrouvés ensemble».$ "Il n'est pas question d'un choix affectif: le "ça" écarte ce qui est à cacher, le lien» remarque la chercheure (Pradelles-Monod, 1999, p. 316).

Tout aussi fréquente, la banalisation constitue un procédé défensif.

Dans une entrevue avec une femme qui a subi deux avortements provoqués et qui tente de persuader la chercheure, autant qu'elle-même, que c'est une situation anodine, elle dit: 
parce que comme intervention c'est rien//sssc'est, bon, une coup' de bonnes crampes pis c'est tout...//Fac euh c'est queqchose qui dure cinq minutes pis euh c'est vraiment pas d'cas faire un gros plat avec ça

L'ensemble de l'entrevue, qui constitue une seule longue dénégation, révèle toute la méfiance de cette jeune femme envers des hommes et sa crainte d'abandon, qui lui fait choisir l'avortement comme solution à ses grossesses. Elle est rassurée parce que

pis, j'ai vu c'que l'on en laisse cc'est un petit bout d'tissu pis euh, ça a pas pantoutt la forme d'un bébé, alors que moi j'm'imaginais que déjà ça avait la forme pis, euh, même pas!

On repère aisément les figures d'exagération (en plus ou en moins), c'est-à-dire les tropes qui recourent à l'amplification ou à l'amoindrissement par le discours. Amplifier peut aussi passer par la gradation - «[... l'autre grand-père il était pas beau, il avait l'air sévère, il avait l'air méchant» (Pradelles-Monod, 1999, p. 314) - ou par l'hyperbole («mon sac pèse une tonne»), qui peut se transmuer en l'ironie «ils adorent faire des pompes».

Parmi d'autres figures, la litote, fréquente, pourrait faire croire à sa proximité avec l'euphémisme; pourtant son effet en diffère, puisqu'elle souligne ce qu'elle semble vouloir cacher, comme dans le "ça ne me dérange pas» d'acceptation québécoise ou le «c'est pas mauvais» d'un gourmand satisfait de son plat. Un pas de plus et l'on a affaire à l'antiphrase, dont le sens exact se déduit du contexte, comme lorsque «ça sent bon ici!» signifie que ça pue.

En fait, quelle que soit le nom du trope, l'attention du chercheur est attirée par des façons de dire évocatrices, par des échappées de la langue.

Lorsque l'une des interlocutrices de Pradelles-Monod dit:

Mon père est mort de tuberculose en 1951//donc à partir de 1950 il était parti en sana [...] mon papa a dîu partir je crois en 1950, fin 50 et il est mort avant Noël 51 (p. 245)

La chercheure remarque que les traces qui manifestent la charge émotionnelle de ce souvenir sont ténues. Il n'en reste pas moins qu'elle entend ce «mon papa», qui dans la bouche d'une adulte traduit l'émotion de la petite fille. 
La psychanalyse a habitué à repérer les dénégations dans le discours qui permettent d'évoquer une idée tout en la niant. La participante à la recherche sur l'avortement provoqué, déjà citée, affirme à propos de son conjoint, qui lui, voudrait avoir des enfants:

[...] parce que bon, euh, l'avortement, c'est pas qu'il est contre...

Dans la recherche de Pradelles-Monod, une représentante de la troisième génération de femmes d'origine espagnole dit:

"je suis/je veux dire je re/je renie pas mes origines//c'est ce que je dis toujours, hein.» En soulignant qu'elle ne les renie pas, elle confirme que celles-ci pourraient être niés (p. 301)

L'annulation rétroactive est proche de la dénégation; ce qui diffère, c'est que la première partie de la phrase contient explicitement l'élément qui va être nié, comme à la planche VI du Rorschach

Feuille, ça ressemble à une feuille, mais ça a pas l'air... (Rossel et al., 2001, p. 114)

Diverses précautions verbales indiquent des tentatives de mise à distance de perceptions, de pensées, des émotions. Par exemple dans le test de T.A.T.:

IV On peut quasiment dire...

IX J'ai l'impression que la femme est morte ici (Dreyfus et Husain, 2001, p. 55)

Dans le registre des articulations discursives, les illogismes de liens par l'usage inadéquat de conjonctions comme «donc», "et», "alors» sont de bons indices, comme relevé dans la recherche d'Ariane Dreyfus et Odile Hussein déjà citée, à la planche II du T.A.T:

On voit dans ses mains les livres, donc elle doit probablement lire, donc s'éloigner des champs, elle est donc dans la solitude...

Cette approche prudente est provoquée par la perception émotionnellement éprouvante de la solitude projetée sur la planche. L’affect pénible est 
finalement nommé, mais il s'agit en même temps d'un certain illogisme: si on s'éloigne des champs, on se retrouve nécessairement seul.

Mais confusions et blocages peuvent aussi se manifester justement parce qu'aucun lien logique ne relie des énoncés hétérogènes. Ainsi, la confusion apparaît chez ce participant à l'une de nos recherches qui désirait être reconnu comme plus savant que l'étudiante chercheure. Au bout d'un développement où il s'est empêtré et a confondu les noms de personnes connues qu'il aurait fréquentées, et après s'en être tiré avec une pirouette, le sujet lui demande:

Vous entendez de [le nom d'un psychologue connu à qui il a fini par se référer]. Il était président du... de [nom de l'association]. Oui !

Question? Question pas claire? En fait, les défauts de la formulation signent un embarras. Qu'exprime ce «entendez» au présent? Manque-t-il une partie de la phrase - «Vous entendez parler de...»? Ce temps présent questionne, lui aussi: faudrait-il qu'elle en entende parler souvent? Quant au "vous ", il s'adresse en principe à la chercheure; mais peut-être implique-t-il l'université à laquelle elle appartient et qu'il a jugée sévèrement? L'hésitation entre «de» et «du» semble indiquer que sa pensée continue à hésiter. Il coupe alors finalement par un «Oui !» qui met fin à la situation embarrassante dans laquelle il s'est mis, alors même qu'il voulait impressionner la chercheure.

\section{En conclusion}

L'expérience de la lecture d'entrevues révèle l'existence d'une multitude d'indicateurs telle qu'il est impossible d'en rendre compte exhaustivement dans le cadre d'un court article. Les émotions s'expriment souvent par onomatopées; diverses tentatives de contrôle du dire reposent sur le clivage entre l'intellect et l'affect: emploi d'abstractions, de formules intellectualisées, de références esthétiques ou symboliques qui visent à fasciner le chercheur; manœuvres de séduction, recherche de complicité par des phrases qui englobent le chercheur dans des propos comme "nous, les femmes...» - toute parole est porteuse de messages directs et indirects.

Aussi cet article ne vise qu'à sensibiliser le lecteur à toutes ces façons d'être qui se communiquent par des façons de dire. En fonction de sa question de recherche, le chercheur peut puiser dans la richesse de manifestations linguistiques, bien au-delà des mots d'esprit, des paradoxes ou des lapsus qui avaient attiré l'attention de Freud. 
En outre, si le sujet qui parle au chercheur dit toujours bien plus que ce qu'il pense communiquer, en même temps l'entrevue est à son service: c'est en parlant qu'une pensée s'élabore. Il est important que le chercheur puisse savoir, en étudiant le texte de l'entrevue, si cette élaboration a bien eu lieu. Cela lui permet d'évaluer la nature (éventuellement traumatique) de ce que le sujet était librement venu lui communiquer à propos de sa question de recherche.

En passer par les mots convoque aussi le passage par des assignations de places, acceptées ou refusées, par une interlocution volontaire et réticente, candide et rusée, stratégique et inconsciente, par l'immédiat de la richesse ou de la pauvreté des mots, du convenu ou du surprenant des récits, des arguments, mais aussi par le temps second de l'analyse qui tâtonne, intuitionne, soupçonne, raisonne... Observation fine, coopération interprétative, perplexité, sagacité, inspiration - aucune recherche qualitative, à plus forte raison d'orientation psychodynamique, voire psychanalytique, ne saurait se passer de ce que donne l'entrevue: la parole du sujet entrevue.

Irène Krymko-Bleton

krymko-bleton.irene@uqam.ca

\section{Notes}

1. Selon l'expression de Marie-Hélène Malandrin, co-fondatrice, avec Françoise Dolto, de la Maison Verte à Paris.

2. «Pape Clément... chevalier Guillaume de Nogaret... roi Philippe... avant un an, je vous cite à paraître au tribunal de Dieu pour y recevoir votre juste châtiment!... Maudits! Maudits! Tous maudits jusqu’à la treizième génération de vos races!» Voir Les Rois maudits de Maurice Druon et le film Les Rois maudits (1972) de Claude Barma.

3. Je présente très brièvement l'évolution de la pensée dans les divers champs d'étude sur la langue en convoquant quelques auteurs-clés. J'indique ainsi le chemin qui m’a menée vers l'idée d'une rencontre possible entre la pragmatique linguistique et la psychanalyse à l'université. Pour un peu plus de précisions, les lecteurs intéressés peuvent se référer à mon article précédent (Krymko-Bleton, 2014) ou directement aux écrits des auteurs mentionnés. Ainsi, la pensée de Ferdinand de Saussure et celle de Charles S. Pierce marquent la fin du $x_{1} x^{e}$ siècle et les débuts du $\mathrm{Xx}^{\mathrm{e}}$. Ils sont suivis de près par Ludwig Wittgenstein dont les écrits datent de la première moitié du $\mathrm{xx}^{\mathrm{e}}$ siècle et par Roman Jacobson qui a publié jusqu'au début des années 80. Jacques Damourette et Édouard Pichon travaillent un peu en autarcie en France dans la première partie du $\mathrm{xx}^{\mathrm{e}}$ siècle. Le philosophe du langage Paul Grice a commencé d'écrire vers la fin des années 50; son influence est allée grandissante jusqu'aux années 80. John Langshaw Austin lui est plus ou moins contemporain. Mikhail Bakhtine, qui avait travaillé en Union Soviétique depuis les années 30, a tardivement été découvert en France où il est publié à titre posthume à partir de la fin des années 70. Finalement, les écrits d'Émile Benveniste, qui a profondément marqué la linguistique française, s'étirent des années 30 aux années 70 . Il 
faut noter que les publications de notes de cours par leurs disciples ou leurs traductions tardives ont participé à promouvoir le travail de certains d'entre eux après leur disparition. Les écrits des auteurs contemporains cités figurent dans les références.

4. Comme François Flahault, présenté plus bas

5, C'est-à-dire l'image que nous construisons de nous-mêmes dans nos échanges. Voir Amossy, 2010.

6. Il est difficile de savoir de quelle valeur parle Lacan puisque les tablettes ou les dés ainsi nommés avaient plusieurs fonctions selon le pays (Grèce antique, Rome, ou même pays celte). Les tessères avaient néanmoins toujours la valeur d'échange.

7. Le concept de «sujet» a depuis rejoint le corpus commun de la psychanalyse française.

8. Comme le notent Jean Laplanche et Jean-Bertrand Pontalis dans Le vocabulaire de la psychanalyse (1997), l'étude de ceux-là a débordé largement, après la publication par Anna Freud de Le Moi et les mécanismes de défense (1936), la question de la gestion des revendications pulsionnelles, en portant désormais sur tout ce qui peut susciter l'angoisse: émotions, situations, exigence du surmoi, etc. Ce repérage de défenses qui se manifestent dans le discours permet l'identification de moments marqués par les conflits psychiques.

9. Les expressions phatiques visent à s'assurer que l'interlocuteur reste attentif («vous m’écoutez?»), comprend le sens de la parole qui lui est adressée («vous comprenez?») et suit l'échange.

10. Pierre Bouthet, Camille Giniès, Hugo Clémot et Perig Cousquer.

11. Trope qui consiste à revenir sur ce que l'on a dit, soit pour l'accentuer, le renforcer, ou au contraire pour l'adoucir. Il peut s'agir autant d'une vraie préoccupation que d'une affectation pour manipuler l'interlocuteur. Ici le sujet revient non pas sur son propre propos mais sur la «proposition» de la chercheure (la planche de Rorschach) pour lui faire une leçon et lui assigner la place de l'élève.

12. Lorsque leur source n'est pas indiquée, les exemples proviennent de mon laboratoire.

13. Selon la Banque de dépannage linguistique de l'Office québécois de la langue française.

\section{Références}

Amossy, R. (2010). La présentation de soi: Ethos et identité verbale. Paris: Presses universitaires de France.

Arrivé, M. (1996). Ce que Lacan retient de Damourette et Pichon: l'exemple de la négation. Langages, 124, 113-124.

Choquet, F. (2001). Le trouble obsessionnel compulsif revisité. Dans O. Husain, C. Merceron et F. Rossel (dir.), Psychopathologie et polysémie: Études différentielles à travers le Rorschach et le TAT (p. 196-215). Lausanne: Payot.

Dreyfus, A. et Husain, O. (2001). Psychopathologie de la dépression: réflexions autour de deux cas Dans O. Husain, C. Merceron et F. Rossel (dir.), Psychopathologie et polysémie: Études différentielles à travers le Rorschach et le TAT (p. 41-61). Lausanne: Payot.

Flahault, F. (1975). La parole intermédiaire, Paris: Seuil.

François, F. (2005). Interprétation et dialogue chez des enfants et quelques autres. Paris: ENS Éditions.

Grice, H. P. (1979). Logique et conversation. Communications, 30, 60-62.

Husain, O. (2005/6). Pertinence diagnostique de la conscience interprétative. Bulletin de psychologie, 480, 627-631.

Husain, O. (2007). Le diagnostic de la perversion à travers le test de Rorschach et le TAT. Dans Tychey, C. (dir.), Clinique des perversions (p. 51-74). Toulouse: Érès. 
Jacques, F. (1982). Anthropologie d'un point de vue relationnel. Paris: Aubier-Montaigne, Jakobson, R. (1963). Essais de linguistique générale. Tome I. Paris: Minuit.

Jakobson, R. (s. d.). Linguistique et poétique. Repéré à http://www.roseaupensant.fr/pages/ textes/textes-sur-le-langage/jacobson-les-6-fonctions-du-message-et-la-fonctionpoetique-en-particulier.html

Krymko-bleton, I. (2014). Rencontre et le discours de la méthode. Filigrane, 23 (2), 109-124. Lacan, J. (1956). Rapport au Congrès de Rome (1953): Fonction et champ de la parole et du langage en psychanalyse. Dans La psychanalyse, 1 (p. 235-322). Paris: Seuil, 1966.

Normand, C. (1985). Les liens du sens. Linx, 13, 125-146.

Pernot, L. (2018). L'art du sous-entendu. Paris: Fayard.

Pradelles-Monod, M.-L. (1999). La construction du lien de filiation entre trois générations de femmes. Repères pour une analyse clinique d'entretiens de recherche. Thèse d'État, Université Louis Pasteur, Strasbourg 1.

Rebourg-roestler, C. (2005/6). Quand le mot devient acte au Rorschach: procédés rhétoriques chez des patients présentant une organisation perverse de personnalité. Bulletin de psychologie, 480, 671-683.

Rossel, F., Merceron, C., Cattin, J.-P. (2001). Dans O. Husain, C. Merceron et F. Rossel (dir.), Psychopathologie et polysémie: études différentielles à travers le Rorschach et le TAT (p. 90-126). Lausanne: Payot.

Roudinesco, E. et Plon, M. (1997). Dictionnaire de psychanalyse. Paris: Fayard.

Matériel non-utilisé de thèses soutenues et en cours de laboratoire d'Irène Krymko-Bleton:

Perrault, M. (2009). Le féminin chez le père en devenir. Étude comparative entre contextes de grossesse avec menace d'accouchement prématuré et grossesse normale (Thèse de doctorat inédite). Université du Québec à Montréal.

Sednaoui, M. (1996). L'alliance paternelle dans le processus du travail d'accueil au cours de la grossesse terminée par un accouchement prématuré pour causes idiopathiques (Thèse de doctorat inédite). Université du Québec à Montréal.

Tissières, N. (thèse en cours). Psychologues de diverses origines face à un matériel multiculturel (dessins d'enfants): analyse des processus interprétatifs (Thèse de doctorat inédite). Université du Québec à Montréal. 